\title{
Cultural transmission and biological markets
}

\author{
Claude Loverdo ${ }^{1}\left([) \cdot\right.$ Hugo Viciana ${ }^{2}(1)$
}

Received: 6 December 2017 / Accepted: 20 October 2018 / Published online: 9 November 2018

(c) Springer Nature B.V. 2018

\begin{abstract}
Active cultural transmission of fitness-enhancing behavior (sometimes called "teaching") can be seen as a costly strategy: one for which its evolutionary stability poses a Darwinian puzzle. In this article, we offer a biological market model of cultural transmission that substitutes or complements existing kin selection-based proposals for the evolution of cultural capacities. We demonstrate how a biological market can account for the evolution of teaching when individual learners are the exclusive focus of social learning (such as in a fast-changing environment). We also show how this biological market can affect the dynamics of cumulative culture. The model works best when it is difficult to have access to the observation of the behavior without the help of the actor. However, in contrast to previous non-mathematical hypotheses for the evolution of teaching, we show how teaching evolves, even when innovations are insufficiently opaque and therefore vulnerable to acquisition by emulators via inadvertent transmission. Furthermore, teaching in a biological market is an important precondition for enhancing individual learning abilities.
\end{abstract}

Keywords Social learning $\cdot$ Comparative advantage $\cdot$ Teaching $\cdot$ Cumulative culture $\cdot$ Partner choice

Electronic supplementary material The online version of this article (https://doi.org/10.1007/s1053 9-018-9649-8) contains supplementary material, which is available to authorized users.

Hugo Viciana

Hugo.Viciana@normalesup.org; Hviciana@iesa.csic.es

Claude Loverdo

claude.loverdo@upmc.fr

1 Laboratoire Jean Perrin, UMR 8237 - CNRS, Sorbonne Universite, 4, place Jussieu, Tour 32-33, 75252 Paris Cedex 05, France

2 Juan de la Cierva Research Fellow, Instituto de Estudios Sociales Avanzados-CSIC, Plaza Campo Santo de los Martires, 7, 14004 Cordoba, Spain 


\section{Introduction}

Cultural transmission is sometimes considered to confer a straightforward advantage unto the group or the individual's kin. However, if the active transmission of culture is such a successful strategy, then where has it occurred in the animal kingdom? Despite the fact that social learning and certain forms of animal traditions are common among many non-human species, active cultural transmission or teaching is a far rarer phenomenon (Boyd and Richerson 1996; Thornton and Raihani 2008).

Strictly speaking, cultural transmission is broadly defined (Viciana 2018). By cultural transmission, one might refer to any process of social learning or the social transmission of artifacts. The set of all types of cultural transmission is huge and variate indeed. It is something that researchers of animal behavior and the evolution of culture are generally well aware of (Hoppitt and Laland 2013, chapter 4). Without being exhaustive, cultural transmission might encompass the process of inadvertent social learning (Danchin et al. 2004) (where one individual reproduces the behavior of another individual when triggered by the observation of some unintended effect of that behavior), processes of cultural communication (Origgi and Sperber 2000), epidemiological processes of cultural attraction (Miton et al. 2015), active cultural transmission or teaching (of which there are different kinds) (Kline 2015), and even cultural niche construction (Odling-Smee et al. 2003).

On the production side of active cultural transmission, "natural pedagogy" (i.e., the dispositions and efforts of adults to make themselves easily understood by children in order to facilitate the transmission of cultural knowledge) is certainly part of the human pattern of cultural transmission (Hewlett et al. 2011). This form of teaching is a good candidate for a universal trait of our species, and perhaps even a biological adaptation (Csibra and Gergely 2009). Such considerations suggest a vertical-transmission view of the evolution of human culture i.e., the direct transmission from a parental generation to its offspring. Nonetheless, another view that is widely accepted among ethnographers claims that adult-infant instruction is rare in hunter-gatherers groups (Atran and Sperber 1991). Moreover, as several case studies in cultural transmission have indicated, non-vertical transmission (the transmission to children from other children or slightly older individuals, as opposed to much older adults) is far more important for cultural transmission than what is often assumed (Aunger 2000; Morin 2015). It has even been argued that non-vertical transmission might constitute a key component of children's and young adults' adoption of much of the cultural repertoire (Harris 1998).

From a population genetics perspective, other considerations also call into question the primacy of vertical transmission in the evolution of culture. Cultural capabilities were plausibly "built for speed" and adaptability (Richerson and Boyd 2000). However, pure vertical cultural transmission is more similar to genetic adaptation than horizontal transmission. Thus, vertical transmission may exhibit properties that make culture adaptive to a lesser extent: vertical 
transmission is more often subject to maladaptive lag and inertia than other forms of cultural transmission (McElreath and Boyd 2008). In changing environments, mother does not always know best. The facilitation of cultural transmission via genetic relatedness, namely as a form of evolved nepotism, conceivably plays a role. However, this role could be easily exaggerated. There are conflicts of interest between parents and siblings (Trivers 1974). In principle, parental manipulation could be selected for, which, in return, could prompt the evolution of devices that counteract the effects of vertical cultural transmission among siblings (Trivers 2011).

Active cultural transmission is fundamentally problematic in light of its costbenefit structure. If what is learned by an individual is so demonstrably useful in terms of fitness that acquiring it makes sense for other individuals, then why bother actively transmitting it? From the standard inclusive fitness perspective of evolution, it follows that traits that do not benefit kin need to benefit their carriers in order to evolve by way of natural selection (Dessalles 2001, 2006). However, in humans a great deal of cultural transmission is directed at non-kin and costly enough to pose a Darwinian puzzle. The question thus remains: why transmit culturally?

The seemingly altruist cultural transmission of fitness-enhancing information yields a free-rider problem that is structured similarly to the standard prisoner's dilemma. Briefly, since active cultural transmission of fitness-enhancing information ("teaching") is a form of cooperation, every individual would be better off if other individuals cooperate, while he or she does not cooperate. Therefore, all else being equal, a population of individuals capable of teaching could be expected to evolve toward a sub-optimal equilibrium: one in which teaching is simply not practiced.

Early on, teaching was characterized by ethologists as a form of biological altruism (Caro and Hauser 1992). In principle, ecological conditions linked to kin selection and alloparentality might have facilitated the evolution of certain cultural capacities (Hrdy 2009; Flinn and Ward 2005). Consequently, the immense majority of formal models that have been used to investigate the evolution of teaching have relied on genetic relatedness in order to explain its stability (Castro and Toro 2014; Fogarty et al. 2011). More recently, Castro et al. (2010) have argued that "humans have developed psychological mechanisms that enable cultural transmission by being receptive to parental advice." Nevertheless, the above mentioned theoretical and empirical considerations largely justify the exploration of complementary, if not alternative, evolutionary pathways through which cultural capacities related to teaching can reach an adaptive equilibrium in a given population [see Sytsma (2012) for a similar argument].

\section{Biological trade and the ecology of social learning}

In this article, we analyze conditions for the evolution of oblique or horizontal active cultural transmission as a behavioral phenotype in a biological market model. Originally proposed by behavioral ecologists Ronald Noë and Peter Hammerstein, biological markets arise when associations between biological individuals are sufficiently uncoerced such that competition occurs not so much by force or its threat, but as a 
need to offer more of what the choosing party "demands." The idea of biological markets thus sheds light on certain selective mechanisms: namely, market effects in which "members of one class can "force" members of another class to evolve traits that would have a negative effect on fitness in the absence of the cooperative interaction" (Noë and Hammerstein 1994, p. 2).

Along with other ecological forces, part of the evolutionary rationale behind active cultural transmission might be the result of biological markets. Models and hypotheses akin to biological markets have already found applications in other arenas of evolutionary psychology, including the psychology of cooperation and mutualism (Frank 1988; Baumard 2010; André and Baumard 2011). To our knowledge, Henrich and Gil-White (2001) first proposed that cultural abilities and knowledge could enter into a market-like exchange of "information goods" and "prestige". Based on previous anthropological observations (Barkow 1975), they formulated a theory in which dominance and prestige hierarchies differ and mix in the context of human hierarchical strategies. In humans, hierarchical status is attainable not only through use of force (i.e., the "dominance" strategy) or power, but also through demonstrations of expertise in certain cultural domains: an ability that, when socially acknowledged, is usually referred to as "prestige" (Cheng et al. 2013). Since status tends to be associated with reproductive success, and since the use of force by way of sheer dominance has probably been selected against during the evolution of our species (Boehm 1999), pursuing competence might have been an advantageous reproductive strategy of primary importance in the history of our species. In what follows, we explicitly incorporate that ecological force into the study of the evolution of cultural transmission.

It is customary to examine the effects of changing environments in the study of cultural evolution. In a very stable environment, a social learner, who has learned a technique from an individual learner, can be copied by another social learner, who can then be copied by another social learner, and so on. In this case, the proportion of individual learners tends to be very low or non-existent (Rogers 1988). When the environment changes very quickly, acquiring older innovations becomes less and less adaptive. Thus, individual learners will be favored, and their population will increase proportionately.

Here, we choose to study another effect: realistically, there is a limit in the number of individuals who can learn from one individual (be it through emulation or active teaching). Additionally, it might be difficult to reproduce the innovative behavior by observation without the help of the actor. Thus, access to expert individuals to learn from can be subject to market forces. We examined two limits. Models 1 and 2 can be understood as ecologies of innovation in which the environment is changing very quickly such that learning innovations from social learners (who have learned from individual learners) introduces too long of an adaptive lag compared to the pace of change in the environment. The other limit is in model 3, which can be understood as a non-changing environment. With no further assumptions, this would lead to a population with a fraction of individual learners tending to 0 . However, we present a scenario that allows for a cumulative culture, including a limited period in an individual's life history where innovation is possible. That can lead to interesting results, which we will discuss. 
Table 1 Abbreviations

\begin{tabular}{ll}
\hline$I$ & Individual learning strategy (with or without teaching) \\
$S$ & $\begin{array}{l}\text { Social learning strategy (either by inadvertent social } \\
\text { transmission or apprenticeship) }\end{array}$ \\
freq $_{X}$ & Frequency of strategy $X$ in the population \\
$W_{X}$ & Fitness of strategy $X$ \\
\hline
\end{tabular}

\section{Model 1: Absence of teaching}

To underscore the necessity of introducing a perspective focused on biological markets, we begin by considering a simple producer/scrounger scenario with frequency dependence that is based on previous attempts to capture basic processes in the evolution of social learning [Boyd and Richerson (2004); for a review, see Aoki and Feldman (2014)]. This model and the subsequent models that we introduce in this article focus on the transmission of adaptive behavioral innovations. As highlighted in the introduction, there are multiple forms of cultural transmission. In terms of interpretation, one can think of models $1-3$ as representing the acquisition of recent behavioral innovations (as opposed to, for instance, the acquisition of "already cultural" traits or traits that derive their adaptive value from the fact that they are shared by others, such as the traits of a specific language or local cultural norms).

We first suppose a minimal case in which there is no active teaching. Agents in the population can follow one of several strategies, each of which has the same baseline fitness, $W_{0}$, in addition to the frequency-dependent fitness based on characteristics of the strategy.

The strategies reproduce in the next generation with probability proportional to the fitness: if at time $T$ there are $n_{I}$ individuals with strategy $I$ of fitness $W_{I}$ (Table 1 ), then the number of individuals with strategy $I$ at time $T+1$ will be $\frac{n_{I} \times W_{I}}{\sum_{j} n_{j} \times W_{j}}$. Such an idealization represents either the result of genetic evolution in an haploid panmictic asexual population or the dynamics resulting from social learning focused on the relative success of other strategies in the population.

In the simplest preliminary form of this scenario, a part of the population follows the strategy of individual learning. Those agents bear a cost, $c$, of learning individually. (This is a common assumption in this type of model. Such a cost could represent either the cost of committing costly errors while learning by oneself, or the opportunity cost of investing time in individual learning instead of something else.) At the same time, the strategy of individual learning also yields a benefit, $b$. To simplify, we suppose here that agents who learn individually always discover an innovation of fitness value, $b$. In Sect. 1 of the Online Appendix, we show that if hiding the innovation is costly, then actively hiding individually acquired innovations is not an evolutionarily stable strategy. Since this finding or discovery is partially observable, it is possible that other agents in the group will attempt to copy the solution by following a social learning strategy. We call agents using this latter strategy "emulators," and the process of social learning without active transmission of fitness enhancing behavior is referred to as "inadvertent social transmission." (One can 
think of emulators as the "scroungers" in the producer/scrounger dynamics of this first model.) In our modelling of the dynamics of individual learners and emulators (models 1 and 2), we will consider the background environment to be changing fast enough such that the value of the previous innovation cancels after each generation. Thus, emulators do not copy other emulators. In model 3, we will study a stationary environment in which social learners can become teachers.

To begin, we assume two conditions: First, only rarely does inadvertent social transmission produce perfect copies of behavior. Emulators who adopt the solution discovered by other agents thus benefit to the degree of $l \times b$ in which $l \leq 1$ is a transformation or "loss factor" associated with social learning (see Enquist and Ghirlanda 2007 on the maladaptiveness of social learning). Second, we suppose that the relative ease of social learning is directly proportional to the number of individual learners (Pagel 2012). In our model, we codify that constraint by imposing a limited number of social learners $N_{e}$ who can learn socially from a given individual learner. That condition is ecologically plausible, at least for a wide range of learning processes used to acquire certain techniques. Furthermore, it is easy to imagine that only a finite number of agents can have access to a given individual learner for the behavior to be adopted. ${ }^{1}$

The average fitness of an agent who learns socially is then dependent on the frequency of those who learn individually according to the following rule:

$$
W_{S}=W_{0}+l \times b \times \min \left(1, N_{e} \times \text { freq }_{I} / \text { freq }_{S}\right)
$$

where fre $_{X}$ is the proportion of strategy $X$ in the population ( $I$ for individual learning, $S$ for social learning), and min denotes a selection of the minimal value between 1 and the effective proportion of emulators that can acquire the behavior given the number of individual learners in the population $N_{e} \times$ fre $q_{I} /$ fre $q_{S}$.

If $N_{e} \times$ freq $_{I}>$ freq $_{S}$, then all emulators can find a model to copy and their fitness will therefore equal $W_{S}=W_{0}+l \times b$. In the opposite case, certain but not all emulators can find a model to learn from. The probability of learning socially is then $N_{e} \times$ freq $_{I} /$ freq $_{S}$. If $l \times b<b-c$, then the individual learning strategy is always more advantageous than the social learning one. At the same time, if $l \times b>b-c$, then the number of social learners will tend to increase until $l \times b \times N_{e} \times$ freq $_{I} /$ freq $_{S}=b-c$, that is, to the point at which both strategies have the same fitness. At that equilibrium, it is the case that:

$$
\text { freq }_{I}=\frac{b-c}{l \times b \times N_{e}+b-c} .
$$

\footnotetext{
1 Mathematically, this condition helps to prevent singularities: without it, a single learning agent suffices in order for all social learners in a large population to be able to acquire the innovation $(N \gg 1)$. However, the number of social learners would abruptly collapse (and become 0 ) when the proportion of individual learners decreases from $1 / N$ to 0 . It is not incoherent to state that social learning is facilitated when the proportion of individual learners in the population is greater.
} 


\section{Model 2: Teaching in a biological market}

\section{Analytical model}

A crucial feature of model 1 is that there is a maximum number $N_{e}$ of emulators that can learn at a given time from one individual learner. At equilibrium, not all emulators have the same kind of access to an individual learner. Indeed, this is the precondition on which the very existence of the market is premised: the individual learners "sell" privileged access to their skills in exchange for biological services, which amounts to "deference" and can take many forms in an informal apprenticeship. To introduce the possibility of teaching, we assume that agents who learn individually — with a frequency in the population freq $q_{I}$ can also follow a strategy by which they actively teach the acquisition of their technique. In addition to the cost of individual learning $c$, such a strategy will have a cost $t$ linked to teaching. As with the previous model, we assume that there is a maximum $N_{a}$ of individuals who can learn from a single teacher as "apprentices" 2 contemporaneously.

Another assumption of our model is that social learners who acquire the technique directly from the teacher will reproduce a perfectly efficacious copy of the teacher's innovation. Although admittedly an idealization (Morin 2016), the point is simply that, for this modality of technological learning, social learning without a teacher sometimes tends to produce a less fit solution than does social learning within the context of a teacher-apprentice relationship. Thus, if there is a teacher, the fitness value of the socially learned technique becomes $b$ instead of $l \times b$. However, individuals who learn socially from a teacher will recompense the teacher via deference mechanisms that have a cost $m$ and that return $m \times g$ to the teacher. It seems reasonable to assume that most of the time $g>1$; however, our model does not strictly depend on that assumption (Table 2).

For deference to evolve in social learning, its cost $m$ must be less than the cost of individual learning $c$. Consequently, at its greatest value $m$ is equal to $c$. For the evolution of teaching, the cost $t$ of teaching must therefore be inferior to $N_{a} \times g \times c$.

Calculating the equilibrium state of the system is not straightforward, as both fre $_{S}$ relative to freq $q_{I}$ and the value of $m$ may evolve. Additionally, it could be that not all the individuals have the same preference $m$.

One method is to examine the evolution of the frequencies and of $m$ independently. We can start assuming that all the individuals in a population have a fixed preference $m$. We can write the fitness values for the teachers and apprentices and obtain their equilibrium frequencies for which their fitnesses are equal (see Sect. 2.1 of Online Appendix). Then, we assume that the frequencies are fixed, the whole population has preference $m$, except that there are mutants. If $N_{a} \times$ freq $_{I}<$ freq $_{S}$, not all apprentices are matched with a teacher, and thus a mutant apprentice with a slightly higher $m$ will be favored, and thus the

\footnotetext{
${ }^{2}$ We make no assumption concerning the specific social configuration of the teacher-apprentice relationship, except that there is some nonzerosumness or collaboration in the basic terms described in the model.
} 
preference $m$ of the apprentices will evolve towards higher values, allowing the preference of the teachers $m$ to also evolve towards higher values. Conversely, if $N_{a} \times$ freq $_{I}>$ freq $_{S}$, not all teachers are paired with $N_{a}$ apprentices, thus $m$ is driven to decrease for the teachers, which then leads to a decrease in $m$ for the apprentices. The next step is to study the effect of the change in $m$ on the frequencies. For the initial $m$, the frequencies were such that apprentices and teachers had the same fitness. If $m$ decreases (respectively increases), then teachers are less (respectively more) fit than apprentices, then the teacher's frequency decreases (resp. increases). Thus the equilibrium point is:

$$
N_{a} \times \text { freq }_{I}=\text { freq }_{S}
$$

which is equivalent to:

$$
\text { freq }_{I}=\frac{1}{1+N_{a}}
$$

and:

$$
m=m_{e q}=\frac{c+t}{N_{a} \times g+1}
$$

in which $m_{e q}$ is the value of $m$ at equilibrium (see more detailed discussion in Sect. 2.2 of the Online Appendix).

By extension, another condition for the evolution of teaching is that $b>m_{e q}$ : an apprentice has to gain more through the acquired technique than the cost of deference. That condition is really constraining for teaching only at very high values of $c$ or $t$. It is most reasonable that $g$ is at least equal to 1 , and $N_{a}$ at least equal to 1 . Therefore, as an example: if $c$ and $t$ remain less costly than $b$, then that condition is fulfilled.

An interesting property of $m_{e q}$ is that it is the $m$ value maximizing the fitness of the population (see Sect. 2.3 of Online Appendix). The evolutionary stable equilibrium is also the state of the system with the highest fitness. In our model, the socalled Rogers' paradox (Aoki and Feldman 2014) does not occur.

We considered here that all social learners have the same preference $m$, with $m>0$, i.e. the social learners reward their teachers. But, there could be a distribution of preferences $m$ in the population, and as in model 1, there could also be social learners (the "emulators") who only try to copy without being taught, provided that $l \times b \geq b-m$ (when $m=m_{e q}(5)$, this condition is equivalent to $\left.l>1-\frac{c+t}{\left(N_{a} \times g+1\right) \times b}\right)$. Interestingly, the presence of these emulators modifies neither the equilibrium between the frequencies of the individual learning and apprentice strategies, nor the evolution of $m$. Even when $l \times b>b-m$, apprentices are not driven to extinction by emulators. In other words, teaching may evolve even if social learning without teaching ("inadvertent social transmission") is still an available and profitable strategy in the population. We can calculate the expected frequency of emulators: their 
Table 2 Parameters and variables of models 1 and 2

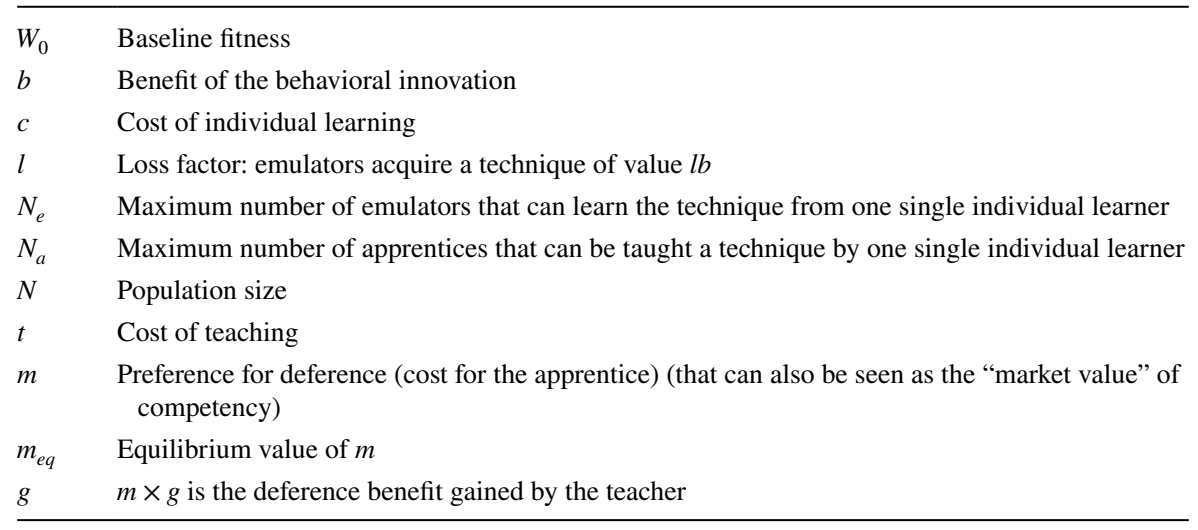

frequency increases until there are not enough individual learners, so that $l \times b \times N_{e} \times$ freq $_{I} /$ freq $_{\text {emul }}=b-m$. This ultimately leads to: ${ }^{3}$

$$
\text { freq }_{I}=\frac{1}{1+N_{a}+\frac{l \times b \times N_{e}}{b-m_{e q}}}
$$

Even if deference is relatively costly, the apprentice strategy can be on par with the emulator strategy, because it enables better access to individual learners who are a source of innovation. In fact, both strategies could still coexist with $l=1$-although the greater the value of $l$, the smaller the frequency of the apprentice strategy. As a result, the assumption that $l<1$ is not necessary.

\section{Simulation}

The analytical calculations assumed mostly homogeneous $m$ preferences, and the evolution of the frequencies and $m$ were considered separately. But both will actually evolve simultaneously, and if $m$ mutates when the strategy is reproduced, the preferences of teachers and apprentices cannot be exactly equal because a mutation towards a slightly higher $m$ for a teacher or a slightly lower $m$ for an apprentice would lead to the inability to enter into a teaching relationship in the first place, thus decreasing the second-generation fitness.

To check that the system converges towards our analytical results, we coded an agent-based simulation. Each individual $j$ is either an individual learner or a social learner, and attributes the reservation value $m_{j}$ to teaching. Random pairs are formed between social learners who have not yet acquired the skill, and individual learners who have not yet taught to $N_{a}$ social learners. If for a given pair, the reservation

\footnotetext{
${ }^{3}$ See more details in Sect. 3 of the Online Appendix.
} 


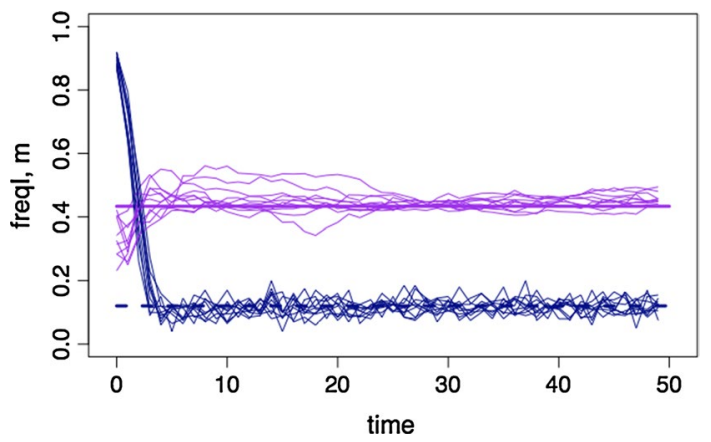

Fig. 1 Simulation example. Frequency of individual learners (dark blue) and mean m values of interactions (light purple) for 10 different simulations, as a function of time (in generations). The horizontal thicker lines represent the predicted values of the frequency of individual learners (dark blue dashed line) and $m$ (light purple solid line). For a population of 200 individuals, with $N_{a}=2, N_{e}=5, W_{0}=0.01$, $b=1, c=0.8, l=1, g=1, t=0.5$, and the typical change in $m$ when transmitting a strategy $\delta m=0.02$. At the beginning of the simulations: $90 \%$ of the population are individual learners, a random $m$ value is attributed to each individual, taken from a uniform distribution between 0 and 1 . (Color figure online)

value $m$ is smaller for the social learner, nothing occurs. But in the opposite case, the individual learner teaches the skill to the social learner at a price $m$ that is taken as the average between the $m$ values of the two individuals. (Any intermediate value between the two values would yield similar results, see supplementary Fig. 14 in Online Appendix.) This method of estimating the actual exchange value of $m$ builds on the idea that there will be some form of bargaining between the two individuals. Pairs are formed until there is no possible additional interaction. Then the population is renewed, with new strategies taken at random proportionally to their fitness in the previous round, and the values $m_{i}$ attributed to teaching in these strategies are copied with small random errors (to allow for the evolution of $m$ ). The frequencies of the different strategies and the average value of $m$ tend to the state defined in Eqs. (5) and (6) (Fig. 1), albeit with fluctuations around these values (see Fig. 2, supplementary Figs. 4-15 in Online Appendix, and Sects. 4 and 5 of Online Appendix). Having validated the results, we can now discuss them.

\section{Results}

As confirmed by the numerical simulations, there are four different regimes, which are summarized in Table 3. Teaching is a stable strategy if the cost of teaching $t$ is smaller than $N_{a} \times g \times c$. Teaching is clearly facilitated when one individual can teach to more apprentices $\left(N_{a}\right)$ (Table 3 and supplementary Fig. 4), when receiving deference provides a higher gain $(g)$ (Table 3 and supplementary Figs. 7 and 11), and if learning the technique individually is costly (c) (Table 3 and supplementary Figs. 5 and 9). Interestingly, this condition does not depend on the characteristics of inadvertent social transmission $\left(N_{e}\right.$ and $l$ ) (Table 3 and Fig. 2B, C). Profiting from "inadvertent social transmission" as emulators do is a stable strategy if the loss in the technique value $(1-l) \times b$ is smaller than the cost 
A

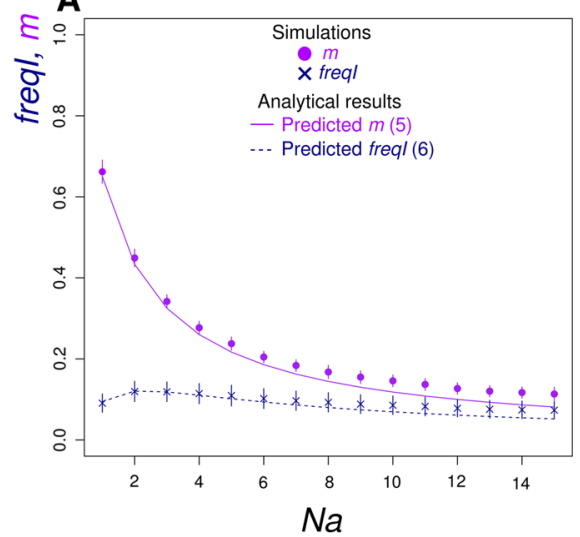

C

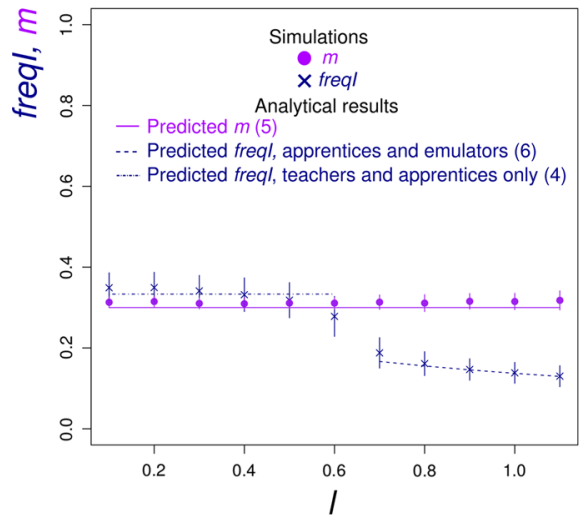

B

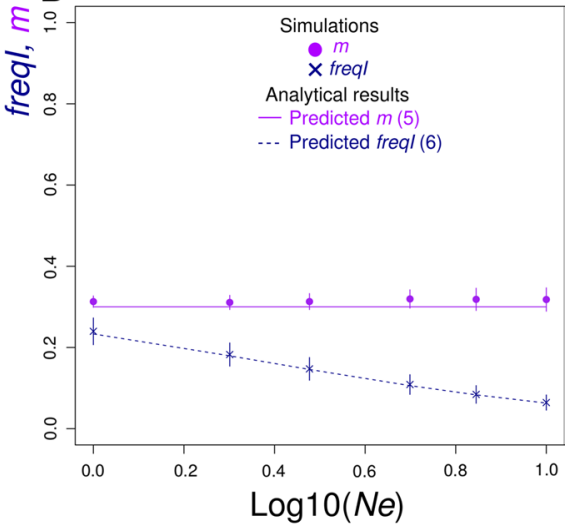

D

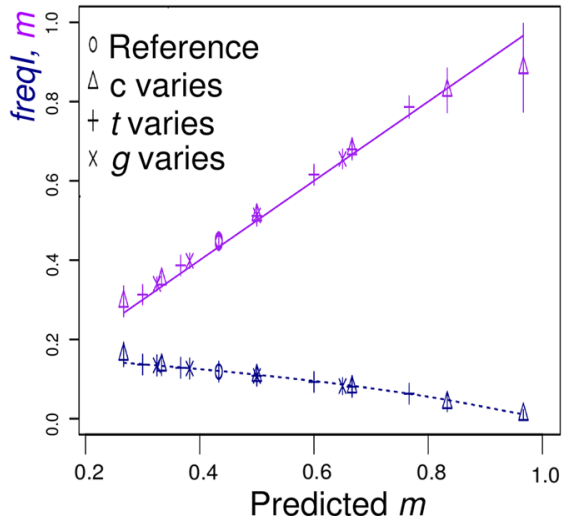

Fig. 2 Dependence of the frequency of individual learners (dark blue) and average value of $m$ in exchanges (light purple) with the different parameters. Results obtained from simulations (symbols) carried out and averaged over 10 simulations, for generations 100 to 200 (see supplementary Fig. 3), and errors-bars represent the standard deviation. Theoretical curves : $m$ (5) (solid purple lines), freq $q_{I}$ with teachers, apprentices, and emulators (6) (dashed blue) or with teachers and apprentices only (4) (dot-dashed blue). For all the simulations, the population is taken as 200 individuals, with the base fitness $W_{0}=0.01$, the technique benefit $b=1$, the typical mutational change on $m \delta_{m}=0.02$, and when the interaction happens, $m$ is taken as the average between the preferences of the two individuals. Initially $50 \%$ of the population are individual learners, with for all individuals, $m$ taken at random between 0 and 1. Except if stated otherwise, the other parameters are $N_{a}=2, N_{e}=3, c=0.8, t=0.5, l=1, g=1$. A Dependence on $N_{a}$. B Dependence on $N_{e}(t=0.1, l=0.9)$. C Dependence on $l(t=0.1)$. For $\mathbf{A}-\mathbf{C}$, freq in simulations is represented by triangles, and $m$ by disks. D We vary $c$ (supplementary Fig. 5), $t$ (supplementary Fig. 6) and $g$ (supplementary Fig. 7). In the regime with both apprentices and emulators, the dependence of $f r e q_{I}$ on $t, g$ and $c$ is predicted to occur only through the value of $m$. Then, instead of representing $m$ and fre $_{I}$ as a function of each of these three parameters separately, we represent them in this panel as a function of the predicted $m$ (5). Supplementary Figs. 5, 6 and 7 show the dependence for each parameter individually. (Color figure online)

of retributing a teacher $\left(m_{e q}\right)$ if there is teaching (Table 3 and Fig. 2C), or smaller than the cost of learning the technique individually $(c)$ if there is no teaching involved (Table 3 and supplementary Fig. 9). 
Table 3 Summary analytical regimes

\begin{tabular}{|c|c|c|}
\hline \multirow[t]{2}{*}{$t<N_{a} \times g \times c$} & $l>1-\frac{m_{e q}}{b}$ & $\begin{array}{l}\text { Teaching and emulating } \\
\text { (5) } m=m_{e q}=\frac{c+t}{N_{a} \times g+1} \\
\text { (6) } \text { freq }_{I}=\left(1+N_{1}+\frac{l \times b \times N_{e}}{b m_{e q}}\right)^{-1}\end{array}$ \\
\hline & $l<1-\frac{m_{e q}}{b}$ & $\begin{array}{l}\text { Teaching } \\
\text { (5) } m=m_{e q}=\frac{c+t}{N_{a} \times g+1} \\
\text { (4) } \text { freq }_{I}=\frac{1}{1+N_{a}}\end{array}$ \\
\hline \multirow[t]{4}{*}{$t>N_{a} \times g \times c$} & $l>1-\frac{c}{b}$ & Emulating \\
\hline & & (2) freq $_{I}=\frac{b-c}{b \times\left(1+l \times N_{e}\right)-c}$ \\
\hline & $l<1-\frac{c}{b}$ & Individual learning only \\
\hline & & $\begin{array}{l}\text { No } m \\
\text { freq }_{I}=1\end{array}$ \\
\hline
\end{tabular}

Table 4 Additional parameters for the cumulative culture model (model 3)

\begin{tabular}{ll}
\hline$\delta b$ & $\begin{array}{l}\text { Improvement in the behavioral skill } \\
\mathscr{C}\end{array}$ \\
$\epsilon$ & $\begin{array}{l}\text { Effort to be invested in improving upon the skill } \\
\text { Probability that the effort at innovating will } \\
\text { lead to an improvement }\end{array}$ \\
\hline
\end{tabular}

In the case of teaching, the value of the deference $m$ at equilibrium increases with $c$, $t$, and decreases with $N_{a}$ and $g$ : deference has to be higher to offset a higher cost of individual learning and teaching, and the higher the number of apprentices per teacher and the higher the factor $g$, the less the deference cost per apprentice (Table 3 and figures). The frequency of the teachers is such that there are $N_{a}$ apprentices per teacher. If there are no emulators, then the teachers' frequency depends only on $N_{a}$. The teachers' frequency is lower when there are emulators and depends on $c, t, g$ only through its dependence on $m$ (Table 3 and Fig. 2D). It decreases with $l$ and $N_{e}$ : the greater the number of emulators, the fewer the teachers and apprentices (Table 3 and Fig. 2B, C). It is maximum for some intermediate value of $N_{a}$ (Table 3 and Fig. 2A).

Another result is that, under a biological market of the type described here, for individual learning to be beneficial, it is sufficient that the cost of individual learning $c$ is smaller than $b \times\left(N_{a} \times g+1\right)-t$, which, except when $t$ is large and $N_{a}$ is small, is likely to be much larger than $b$. Hence, there are investments in skills for which benefits would not be sufficient in themselves, which thus become attractive because of the extra incentive linked to the sociability of teaching.

Our model assumed that apprentices do not become teachers. There are two main reasons for which this hypothesis can be valid for certain technological skills. One is if teaching a technique requires a deeper level of understanding that can be acquired by individual learning only. For instance, most university teaching is done by people who are also researchers, partly because it is thought that complex notions are more efficiently taught by individuals having 
an understanding of them deeper than the base level needed to merely use them. The other reason is in the case of the environment changing fast enough such that the techniques become obsolete, thus leaving an inadequate amount of time for second-hand teaching. If it was possible for apprentices to become teachers later on, the model would need to take into account a more complex time dimension and study the interaction of the time scales of changes in the environment versus the amount of time needed for individual learning, emulation, apprenticeship, and the integration of the fitness over the lifetime of an individual. In our next model, we allow for the possibility that apprentices become teachers.

\section{Model 3: Cumulative culture}

Previous research has shown that social learning per se does not automatically lead to cumulative culture, that is, sustained evolution of ever increasingly adaptive cultural techniques (Enquist and Ghirlanda 2007). In model 2, we have shown that the market for deference supports the increased costs of innovations. Accordingly, we believe that taking those sorts of biological markets seriously can shed light on ecological forces active in the evolution of cumulative culture.

Until now, we have considered the skill to be fixed. Here, however, we consider a different model, in which the skill of value $b$ can be improved by an increment $\delta b$ with probability $\epsilon$ when effort $\mathscr{C}$ is invested into innovation (Table 4 ). We consider that at each time step, a new individual enters the population of size $N$, whereas the "oldest" individual dies. The entrance can represent either a birth-more realistically a child's coming of age and being prepared for the apprenticeship —or a migration. If there is no active teaching, then the new individual copies the best skill in the population, of value $b(T)$; however this is achieved imperfectly. The individual will thus have a skill of value $l \times b(T)$, in which $T$ represents the moment in time when the technique is copied by the newly arrived agent. We assume that individuals can recognize the best skill, and access the value of parameters $\mathscr{C}, \delta b$ and $\epsilon$. We also assume that innovation can occur only during the period when the new individual enters the group and acquires its skill. This latter assumption represents the idea that some periods are more prone to innovation than others. The new individual can decide whether to invest in innovation depending on whether $\epsilon \times \delta b>\mathscr{C}$. If $\epsilon \times \delta b<\mathscr{C}$, then no innovation is ever made, and, provided that $l<1$, the skill will be completely lost in the population over time. If $\epsilon \times \delta b>\mathscr{C}$, the skill will be improved upon by $\delta b$ per each $1 / \epsilon$ new individuals on average. Population size matters (Kline and Boyd 2010): if $1 / \epsilon \gg N$, then the innovations will not occur often enough to preserve the skill in the population. If $1 / \epsilon \ll N$, then the value of the skill over time will tend toward the point at which the imperfect copy and the innovation compensate: $b=\delta b /(1-l)$. A more detailed discussion can be found in Sect. 6 of the Online Appendix.

For active teaching, when the new individual enters the population, many potential teachers are available, meaning that there will be active teaching as long as $m \times g>t$. Due to competition among teachers, $m$ will tend to $t / g$. If $t / g<(1-l) \times b$, then the 
new individual will prefer to learn the technique via active teaching instead of emulation, and thus end up learning the best skill $b$ of the population. At that point, when choosing whether to invest in innovation, the individual will compare the investment cost $\mathscr{C}$ not only with the direct benefit $(\epsilon \times \delta b)$, but also the direct benefit plus the benefit expected from teaching the innovation to the rest of the population. Since the new individual has a monopoly on the skill (assuming that time constraints make that all the individuals in the population want to acquire the technique as soon as possible, rather than waiting for second-hand teaching from apprentices), other individuals in the population will tend to reward his or her teaching by a maximum of at least $m=(1-l) \times(b+\delta b)$. As a result, the expected benefit from teaching is $\min \left(N, N_{a}\right) \times g \times(1-l) \times(b+\delta b)-t$ (see Sect. 7 of Online Appendix $)$.

In summary, populations with active teaching differ from those with only inadvertent social learning in two ways. First, because the skill can be learned more accurately, cumulative innovations are facilitated and the value of the skill can continue to increase. Second, innovation is favored because its benefits might also derive from deference and prestige. Accordingly, in biological markets, evolved teaching has the double effect of both promoting cumulative culture and, most importantly, enhancing individual learning.

\section{Discussion and conclusions}

Modeling evolutionary social dynamics offers proof of the internal consistency of hypothesized evolutionary selective pressures (McElreath and Boyd 2008). Thus, the models presented here corroborate the logical soundness of some intuitions previously formulated in purely verbal arguments (Henrich and Gil-White 2001, see "Introduction" section). They also provide a well-articulated mathematical framework that addresses the current dearth of models for the evolutionary milestone that is the evolution of teaching (Kline 2015).

Reciprocity-based models are not usually well-equipped to accommodate the kinds of hierarchies and asymmetries that we describe in model 2. Furthermore, reciprocity-based cooperation models usually focus more on turn-taking and the partner control aspect of repeated interactions than on partner choice, outside options, and active discrimination. We have shown that market effects can account for relevant dimensions of the sociability of teaching, such as the propensity to transmit fitness-enhancing information, as well as the evolution of deference. We believe that these important aspects of human social learning are better studied by focusing on the supply and demand demographic dynamics of a biological trade, rather than on the standard reciprocity mechanism.

We have provided a partner-choice model for the evolution of teaching, which focuses on the functional aspects of teacher-apprentice cooperation. This account does not offer an exhaustive evolutionary characterization of the emergence of teaching. Teaching is, after all, a complex ethological category that subsumes differentand presumably related - types of phenomena (Kline 2015). Moreover, the models presented are not intended to be so much of a realistic depiction of the actual evolutionary process as an exploration of general ecological conditions for the evolution 
of teaching. However, our work nevertheless points to possible evolutionary pathways, which, one can only surmise, have received little attention because they have not been mathematically modeled. One such possible paleoanthropological pathway is that the structure of communication and nonzerosumness inherent in the form of the basic apprenticeship system described here might have preceded (instead of followed) the evolutionary emergence of modern (i.e., Middle Paleolithic) human inventiveness (McBrearty and Brooks 2000).

We have additionally shown that teaching can evolve under certain conditions. First, individual learning or learning without relying on others' experience is costly. Second, certain techniques are constrained in terms of the number of individuals who can socially learn the technique from a single expert. Under those conditions, demographic dynamics could force social learners, who want to acquire the adaptive behavior discovered by individual learners, to pay a price in the form of deference. Furthermore, although it is unnecessary, the evolution of teaching is facilitated for learning certain techniques if social learning without explicit teaching_-"eavesdropping" (Danchin et al. 2004)_yields imperfect copying in which adaptive value can be lost. Crucially, genetic relatedness and parent-offspring nepotism (Castro and Toro 2004) are not strictly necessary either.

An important observation that emerges from our work is that evolved teaching might be the mother of invention. In other terms, natural pedagogy and communication skills may precede, but not necessarily follow, the appearance of complex forms of culture. This dynamic runs counter to the perspective sometimes advanced, which holds that teaching evolved for novices as a response to increasingly complex “opaque" cultural forms (Caldwell 2015; Gergely and Csibra 2006). In keeping with this evolutionary hypothesis, complex cumulative culture necessarily preceded evolved teaching. However, as we show here, teaching might constitute an evolutionarily stable strategy, even if the existing cultural forms are not opaque enough for novices. Thus, teaching could evolve when inadvertent social transmission (i.e., social learning without teaching) remains a thriving strategy in the population.

Undoubtedly, access to various forms of social learning cannot be controlled in such a way as to give rise to biological exchange markets: "eavesdropping" or inadvertent social facilitation could be the most frequent form of social learning in nature (and perhaps even in humans). Nevertheless, in humans, important forms of technique acquisition can be reasonably controlled and even monopolized to some extent. For instance, ethnographic studies of stone-tool production (Stout et al. 2002) confirm that the adult acquisition of certain sophisticated skills can be perceived as a form of transferable intellectual property. Such a capacity for transmission is endowed with a form of authority that is often safeguarded and administered in a teacher-apprentice system via manifestations of personal commitment. In more modern settings, partner choice has widely been observed to be crucial in acquiring competence within organizations (Blau 1964).

In contrast to non-human social learning, certain forms of human social learning are characterized by both the sophistication of cognitive mechanisms at work and the important constitutive role played by collaboration and nonzerosumness. These characteristics eventually give rise to apprenticeship structures (Waal 2001; Sterelny 
2012). In this article, we have shown how those behavioral strategies can attain evolutionary equilibrium and persist in a population.

Naturally, not all forms of cultural diffusion rely on competence-based partner choice, a point that can hardly be overemphasized. However, some forms of human social learning depend far more on competence-based partner choice than others-a fact that helps explain the existence of several interesting regularities in the human psychology of competence assessment, admiration, and deference (Fessler 2006).

At the proximate level, hierarchical tendencies of this sort are not entirely specific to humans. In fact, other animals have been observed to behave in ways consistent with the predictions of biological markets. In particular, non-human primates, such as chimpanzees (Pan troglodytes), have demonstrated an ability to discriminate possible partners based on their abilities (Melis et al. 2006). Moreover, experimental studies have shown how different species of primates can temporarily adjust their hierarchical behavior after individuals in their group have acquired some valuable cultural competence [see Stammbach (1988) for interesting work on Rhesus macaques]. Grooming behavior has been shown to adapt to the supply and demand characteristics of a biological market in which at least one individual in a group has learned to use a tool in order to obtain a valuable, shareable food (Fruteau et al. 2009). Indeed, it has even been suggested that, for some species, grooming could be a form of proto-currency in primate exchange markets (Barrett and Henzi 2006).

In humans, considerable evidence points to the existence of both competence assessment and prestige-signaling behavior, the latter being a form of communicating that one excels in a given domain (Tracy and Matsumoto 2008). Although the human ability to detect competence in a given domain is certainly far from perfect (Mauboussin 2012), it nevertheless functions as a satisfying heuristic in many settings. Competence is assessed through both fast and slow processes of cognition. At its most rapid rate, the adult judgment of competence can be made in as little as 100 milliseconds, and those judgments are sometimes highly persistent and difficult to override (Fiske et al. 2007). Early on, children also begin to pay special attention to individuals judged as competent in a given domain (Keil et al. 2008). The current consensus maintains that children commonly use two different pathways to judge the reliability of an informant: one related to trust and benevolence and the other related to competence and ability (Mascaro and Sperber 2009; Harris 2012).

In models 2 and 3, we have included relevant characteristics associated with the sociability of certain forms of active cultural transmission. However, we have only scratched the surface of what biological trade models could offer in terms of modeling social learning dynamics. It would be interesting to further explore the evolutionary dynamics linked to maladaptive biases related to the human psychology of competence and prestige detection. For instance, we did not explore here the complex dynamics that could follow if social learners were to adopt the techniques and behaviors of other social learners, who are no longer tracking the environment through individual learning and innovation, but who might receive some form of social reward due to further transmitting a highly prized form of "knowledge," regardless of whether that knowledge proves to be ineffectual or of little direct use. Moreover, the amount of effort that cultural mentors invest in teaching their 
apprentices even when they are not genetically related to them, the diminishing fitness values of the technology if shared, or the reliability of the deference provided by the apprentices are all interesting features for which genetic conflict and partner choice could be fruitfully modeled. We hope to encourage further work in this area.

Regarding important aspects of the evolution of cultural transmission, we have suggested that the partner-choice framework (Nesse 2009) is better equipped than other theoretical frameworks that rely exclusively on either partner control or nepotistic genetic relatedness (Noë and Voelkl 2013). The free-rider problems linked to fitness-enhancing cultural transmission, along with cultural parental manipulation, are largely by-passed by evolutionary systems, such as those described in this article. Nearly a century ago, Lev Vigotsky characterized human social learning as an eminently cooperative activity. Biological market models can incorporate the nonzerosumness of human social learning, account for findings related to the anthropology of deference and prestige, and reveal surprising evolutionary processes that lead to cumulative culture.

\section{Data availability}

An Online Appendix including further analytic details, supplementary figures, and simulation code is provided.

Acknowledgements HV received support from a La Caixa Foundation Scholarship at the initial stage of the preparation of this work. This article has benefited from feedback of audiences at the University of Cambridge, the University of Granada, the University of Louvain-la-neuve, and the University of Paris 1. Special thanks should be given to Camilo Cela-Conde, Jean Gayon, Gabi Lipede, Pierre Livet, Hugo Mercier, Susana Monso, Dan Sperber, Neftali Villanueva, and several anonymous reviewers for comments on earlier versions of this work. In remembrance of Jean Gayon (1949-2018).

\section{References}

André JB, Baumard N (2011) The evolution of fairness in a biological market. Evolution 65(5):1447-1456

Aoki K, Feldman MW (2014) Evolution of learning strategies in temporally and spatially variable environments: a review of theory. Theor Popul Biol 91:3-19

Atran S, Sperber D (1991) Learning without teaching: its place in culture. In: Annual workshop on culture, schooling and psychological development, 4th June 1987, Tel Aviv U, Ramat Aviv, Israel, Ablex Publishing

Aunger R (2000) The life history of culture learning in a face-to-face society. Ethos 28:445-481

Barkow JH (1975) Prestige and culture: a biosocial interpretation. Curr Anthropol 16:553-572

Barrett L, Henzi SP (2006) Monkeys, markets and minds: biological markets and primate sociality. In: Kappeler PM, van Schaik CP (eds) Cooperation in primates and humans. Springer, Berlin, pp 209-232

Baumard N (2010) Comment nous sommes devenus moraux: une histoire naturelle du bien et du mal. Odile Jacob, Paris

Blau PM (1964) Exchange and power in social life. Transaction Publishers, Piscataway

Boehm C (1999) Hierarchy in the forest: egalitarianism and the evolution of human altruism. Harvard University Press, Cambridge

Boyd R, Richerson PJ (1996) Why culture is common, but cultural evolution is rare. Proc Br Acad 88:77-94 
Boyd R, Richerson PJ (2004) The origin and evolution of cultures. Oxford University Press, Oxford

Caldwell CA (2015) Experimental studies of cumulative culture in modern humans: what are the requirements of the ratchet? In: Mesoudi A, Aoki K (eds) Learning strategies and cultural evolution during the Palaeolithic. Springer, Tokyo, pp 145-154

Caro TM, Hauser MD (1992) Is there teaching in nonhuman animals? Q Rev Biol 67:151-174

Castro L, Toro MA (2004) The evolution of culture: from primate social learning to human culture. Proc Natl Acad Sci USA 101(27):10,235-10,240

Castro L, Toro MA (2014) Cumulative cultural evolution: the role of teaching. J Theor Biol 347:74-83

Castro L, Castro-Nogueira L, Castro-Nogueira MA, Toro MA (2010) Cultural transmission and social control of human behavior. Biol Philos 25(3):347-360

Cheng JT, Tracy JL, Foulsham T, Kingstone A, Henrich J (2013) Two ways to the top: Evidence that dominance and prestige are distinct yet viable avenues to social rank and influence. J Personal Soc Psychol 104(1):103

Csibra G, Gergely G (2009) Natural pedagogy. Trends Cogn Sci 13(4):148-153

Danchin E, Giraldeau LA, Valone TJ, Wagner RH (2004) Public information: from nosy neighbors to cultural evolution. Science 305(5683):487-491

Dessalles JL (2001) Why we talk: the evolutionary origins of language. Oxford University Press, Oxford

Dessalles JL (2006) Le langage humain à la lumière de l'évolution. In: Actes des XXVIes journees d'etude sur la parole

Enquist M, Ghirlanda S (2007) Evolution of social learning does not explain the origin of human cumulative culture. J Theor Biol 246(1):129-135

Fessler DM (2006) Steps toward an evolutionary psychology of a culture-dependent species. In: Carruthers P, Laurence S, Stich S (eds) The innate mind, vol 2. Oxford University Press, Oxford, pp $61-77$

Fiske ST, Cuddy AJ, Glick P (2007) Universal dimensions of social cognition: warmth and competence. Trends Cogn Sci 11(2):77-83

Flinn MV, Ward CV (2005) Ontogeny and evolution of the social child. In: Ellis BJ, Bjorklund DF (eds) Origins of the social mind: evolutionary psychology and child development. The Guilford Press, New York, pp 19-44

Fogarty L, Strimling P, Laland KN (2011) The evolution of teaching. Evolution 65:2760-2770

Frank RH (1988) Passions within reason: the strategic role of the emotions. W. W. Norton \& Co, New York

Fruteau C, Voelkl B, Van Damme E, Noë R (2009) Supply and demand determine the market value of food providers in wild vervet monkeys. Proc Natl Acad Sci 106(29):12,007-12,012

Gergely G, Csibra G (2006) Sylvia's recipe: the role of imitation and pedagogy in the transmission of cultural knowledge. In: Enfield NJ, Levenson SC (eds) Roots of human sociality: culture, cognition, and human interaction. Wiley, New York

Harris JR (1998) The nurture assumption: why children turn out the way they do. Simon and Schuster, New York

Harris PL (2012) Trusting what you're told: how children learn from others. Harvard University Press, New York

Henrich J, Gil-White FJ (2001) The evolution of prestige: freely conferred deference as a mechanism for enhancing the benefits of cultural transmission. Evolut Hum Behav 22(3):165-196

Hewlett BS, Fouts HN, Boyette AH, Hewlett BL (2011) Social learning among Congo Basin hunter gatherers. Philos Trans R Soc Lond B Biol Sci 366(1567):1168-1178

Hoppitt W, Laland KN (2013) Social learning: an introduction to mechanisms, methods, and models. Princeton University Press, Princeton

Hrdy SB (2009) Mothers and others: the evolutionary origings of mutual understanding. Harvard University Press, Cambridge

Keil FC, Stein C, Webb L, Billings VD, Rozenblit L (2008) Discerning the division of cognitive labor: an emerging understanding of how knowledge is clustered in other minds. Cogn Sci 32(2):259-300

Kline MA (2015) How to learn about teaching: an evolutionary framework for the study of teaching behavior in humans and other animals. Behav Brain Sci 38:1-71. https://doi.org/10.1017/S0140 525X14000090

Kline MA, Boyd R (2010) Population size predicts technological complexity in Oceania. Proc R Soc Lond B Biol Sci 277(1693):2559-2564

Mascaro O, Sperber D (2009) The moral, epistemic, and mindreading components of children's vigilance towards deception. Cognition 112(3):367-380 
Mauboussin MJ (2012) The success equation: untangling skill and luck in business, sports, and investing. Harvard Business Press, New York

McBrearty S, Brooks AS (2000) The revolution that wasn't: a new interpretation of the origin of modern human behavior. J Hum Evolut 39(5):453-563

McElreath R, Boyd R (2008) Mathematical models of social evolution: a guide for the perplexed. University of Chicago Press, Chicago

Melis AP, Hare B, Tomasello M (2006) Chimpanzees recruit the best collaborators. Science 311(5765):1297-1300

Miton H, Claidière N, Mercier H (2015) Universal cognitive mechanisms explain the cultural success of bloodletting. Evolut Hum Behav 36(4):303-312

Morin O (2015) How traditions live and die. Oxford University Press, Oxford

Morin O (2016) Reasons to be fussy about cultural evolution. Biol Philos 31(3):447-458

Nesse RM (2009) Runaway social selection for displays of partner value and altruism. In: Verplaetse J, Schrijver J, Vanneste S, Braeckman J (eds) The moral brain. Springer, Dordrecht, pp 211-231

Noë R, Hammerstein P (1994) Biological markets: supply and demand determine the effect of partner choice in cooperation, mutualism and mating. Behav Ecol Sociobiol 35(1):1-11

Noë R, Voelkl B (2013) Cooperation and biological markets: the power of partner choice. In: Sterelny K, Joyce R, Calcott B, Fraser B (eds) Cooperation and its evolution. The MIT Press, Cambridge, pp $131-151$

Odling-Smee J, Laland KN, Feldman MW (2003) Niche construction: the neglected process in evolution. Princeton University Press, Princeton

Origgi G, Sperber D (2000) Evolution, communication and the proper function of language. In: Carruthers $\mathrm{P}$, Chamberlain A (eds) Evolution and the human mind: language, modularity and social cognition. Cambridge University Press, Cambridge, pp 140-169

Pagel M (2012) Wired for culture: origins of the human social mind. W. W. Norton \& Co, New York

Richerson PJ, Boyd R (2000) Built for speed: pleistocene climate variation and the origin of human culture. In: Tonneau F, Thompson NS (eds) Perspectives in ethology. Springer, Boston, pp 1-45

Rogers AR (1988) Does biology constrain culture? Am Anthropol 90(4):819-831

Stammbach E (1988) Group responses to specially skilled individuals in a Macaca fascicularis group. Behaviour 107(3):241-266

Sterelny K (2012) The evolved apprentice. MIT Press, Cambridge

Stout D, Bril B, Roux V, DeBeaune S, Gowlett JAJ, Keller C, Wynn T, Stout D (2002) Skill and cognition in stone tool production: an ethnographic case study from Irian Jaya. Curr Anthropol 43(5):693-722

Sytsma J (2012) Information supply and demand: resolving Sterelny's paradox of cultural accumulation. In: Payette N, Hardy-Vallee B (eds) Connected minds: cognition and interaction in the social world. Springer, pp 212-223

Thornton A, Raihani NJ (2008) The evolution of teaching. Anim Behav 75(6):1823-1836

Tracy JL, Matsumoto D (2008) The spontaneous expression of pride and shame: evidence for biologically innate nonverbal displays. Proc Natl Acad Sci 105(33):11,655-11,660

Trivers R (2011) The folly of fools: the logic of deceit and self-deception in human life. Basic Books, New York

Trivers RL (1974) Parent-offspring conflict. Am Zool 14(1):249-264

Viciana H (2018) Animal behavior research and the concept of culture. Manuscript submitted for publication

Waal F (2001) The Ape and the Sushi Master. Allen Lane, London 